\title{
Conocimiento sobre cuidados paliativos pediátricos de los pediatras de atención primaria del Principado de Asturias, España
}

\author{
Vanessa Moya-Dionisio* \\ Centro de Salud Naranco, Oviedo, Principado de Asturias, España
}

\begin{abstract}
Resumen
Introducción: Es importante conocer y evaluar la experiencia y el conocimiento de los pediatras de atención primaria del Principado de Asturias (España) sobre los cuidados paliativos pediátricos. Métodos: Se llevó a cabo un estudio descriptivo y transversal mediante un cuestionario dirigido a los pediatras de atención primaria del Principado de Asturias entre mayo y junio de 2018. Resultados: El 77\% de los participantes no poseía formación previa y el 100\% consideró que sus conocimientos en cuanto al tema no eran adecuados, a pesar de que el 37\% había atendido en alguna ocasión a pacientes subsidiarios de este tipo de cuidados. Casi la totalidad de los encuestados (97\%) consideraron necesaria la creación de una unidad de cuidados paliativos pediátricos. Conclusiones: El conocimiento del pediatra de atención primaria del Principado de Asturias con respecto a los cuidados paliativos pediátricos es muy escaso en una región en la que no se dispone de una unidad pediátrica exclusiva. Sería interesante aprovechar la gran disposición de este grupo de profesionales para mejorar su formación, además de incorporar la materia en facultades y en la formación del médico interno residente.
\end{abstract}

Palabras clave: Atención primaria. Cuidados paliativos pediátricos. Encuesta. Mortalidad.

\section{Knowledge of primary care pediatricians about pediatric palliative care in the Principality of Asturias, Spain}

\begin{abstract}
Background: To know and assess the experience and knowledge among primary care pediatricians about pediatric palliative care in the Principality of Asturias (Spain). Methods: A descriptive and cross-sectional analysis was conducted using a survey among primary care pediatricians in the Principality of Asturias between May and June 2018. Results: The majority of participants (77\%) did not receive previous training, and $100 \%$ considered that their knowledge on the subject was insufficient, although $37 \%$ had occasionally attended to palliative care patients. Almost all participants (97\%) considered that a pediatric palliative care unit is necessary. Conclusions: The knowledge of primary care pediatricians about pediatric palliative care is deficient in the Principality of Asturias, a region where no exclusive pediatric unit exists. It would be interesting to seize the opportunity to improve the training of these pediatricians, given their great willingness, and to incorporating the subject into colleges and medical intern formation as well.
\end{abstract}

Key words: Primary health care. Pediatric palliative care. Survey. Mortality.

\section{Correspondencia:}

*Vanessa Moya Dionisio

E-mail: vanemd@ hotmail.es
Fecha de recepción: 24-02-2020

Fecha de aceptación: 25-04-2020

DOI: 10.24875/BMHIM.20000046
Disponible en internet: 17-07-2020 Bol Med Hosp Infant Mex. 2020;77(4):195-201

www.bmhim.com 1665-1146/C 2020 Hospital Infantil de México Federico Gómez. Publicado por Permanyer. Este es un artículo open access bajo la licencia CC BY-NC-ND (http://creativecommons.org/licenses/by-nc-nd/4.0/). 


\section{Introducción}

La Organización Mundial de la Salud (OMS) define los cuidados paliativos pediátricos (CPP) como aquellos cuidados basados en mejorar la calidad de vida de los niños que se enfrentan a enfermedades potencialmente mortales y de sus familias, a través de la prevención y el alivio del sufrimiento, identificando y tratando de forma precoz el dolor y otros problemas, tanto físicos como emocionales, sociales y espirituales ${ }^{1}$.

Los CPP se deben proporcionar de forma continua, integral e individualizada por parte de un equipo multidisciplinario, comenzar cuando se diagnostica una enfermedad amenazante para la vida y continuar independientemente de que el niño reciba o no tratamiento activo para la propia enfermedad ${ }^{2}$.

Tras detectar que los recursos para estos pacientes eran más bien inexistentes se están desarrollando equipos especializados, ya que el interés por la formación en CPP para ofrecer estos cuidados de forma igualitaria y plena a la sociedad es evidente ${ }^{3}$. A pesar de este crecimiento exponencial, los recursos proporcionados aún no son suficientes ${ }^{4,5}$. En España, aunque ha habido un avance en el número de recursos de CPP (de tres en 2009 a nueve en 2016) y se identifican recursos pediátricos en ocho comunidades autónomas y múltiples iniciativas, es indudable que todavía queda mucho por hacer ${ }^{6}$.

Para minimizar las diferencias, en ocasiones es necesaria la colaboración de otros profesionales no especializados en la materia, entre los que se encuentra el pediatra de atención primaria, quien puede ser un buen colaborador dentro del equipo multidisciplinario que controla el proceso del niño, ya que se trata de una persona cercana con conocimientos previos del diagnóstico del paciente, y además constituye una figura accesible y de confianza?.

La Estrategia de Cuidados Paliativos para Asturias (2009) establece que la población diana serán las personas en situación de enfermedad avanzada - con pronóstico de vida limitado- y sus familias, pero no refiere de forma específica ninguna necesidad básica para la atención a los niños con enfermedades limitantes para la vida ${ }^{8}$.

En el momento de la realización de este estudio no se disponía de dispositivos exclusivos para la población pediátrica en el Principado de Asturias. Sin embargo, actualmente se encuentra en marcha la creación de una unidad específica de CPP.

El objetivo de este estudio fue conocer y evaluar la experiencia y el conocimiento de los pediatras de atención primaria sobre los CPP en el Principado de Asturias.

\section{Métodos}

Se llevó a cabo un estudio descriptivo transversal mediante un cuestionario dirigido a todos los pediatras de atención primaria $(n=85)$ del Principado de Asturias, entre los meses de mayo y junio de 2018.

El cuestionario se creó a través de la página https:// docs.google.com/document/u/0/ para ser completado de forma anónima y voluntaria. Se envió la dirección de enlace para acceder mediante el correo electrónico y se adjuntaron el consentimiento informado y un documento explicativo sobre el objetivo del estudio (además de una dirección electrónica para consultar dudas).

El cuestionario estaba formado por 16 preguntas y dividido en tres secciones. En la primera parte se solicitaban datos demográficos, como sexo, edad, lugar de trabajo y años de ejercicio como pediatra de atención primaria. La segunda parte estaba dedicada a conocimientos y conceptos sobre CPP y la experiencia personal en este ámbito. La primera pregunta de este apartado era una pregunta abierta: «¿Cómo definiría el concepto de cuidados paliativos?». Para el análisis de esta pregunta se evaluó cada una de las respuestas y se realizó una categorización inicial tras observar las respuestas similares o más comunes. Posteriormente, se determinó la frecuencia de las respuestas y se clasificaron. La tercera sección se centraba en la propia percepción del grado de formación de los participantes y el interés por mejorar sus habilidades y conocimientos (Tabla 1).

Una vez recibidas las respuestas, la información se incorporó a la base de datos, y estos se analizaron mediante Excel 2007. La descripción de los datos cualitativos se hizo como frecuencias absolutas $y$ porcentajes.

\section{Resultados}

De los 85 pediatras de atención primaria a los que se envió la encuesta, la contestaron 35 (41.2\%). El 83\% de los encuestados era de sexo femenino, con una media de edad de 48 años, y el $68 \%$ trabajaba en un medio urbano. Casi el $66 \%$ contaba con más de 10 años de experiencia como pediatras de atención primaria; de estos, el 51\% llevaba más de 20 años ejerciendo en el área.

Como respuesta a la pregunta abierta «¿Cómo definiría el concepto de cuidados paliativos?», la mayoría 
Tabla 1. Encuesta y respuestas obtenidas

\begin{tabular}{|c|c|c|}
\hline Variable & Frecuencia (n) & Porcentaje \\
\hline Edad en años* & 48 & 29-62 \\
\hline $\begin{array}{l}\text { Sexo } \\
\text { Masculino } \\
\text { Femenino }\end{array}$ & $\begin{array}{c}6 \\
29\end{array}$ & $\begin{array}{l}17 \\
83\end{array}$ \\
\hline $\begin{array}{l}\text { Lugar de trabajo } \\
\text { Urbano } \\
\text { Rural }\end{array}$ & $\begin{array}{l}23 \\
11\end{array}$ & $\begin{array}{l}68 \\
32\end{array}$ \\
\hline $\begin{array}{l}\text { Años de ejercicio en la pediatría } \\
<5 \text { años } \\
5-10 \text { años } \\
10-20 \text { años } \\
>20 \text { años }\end{array}$ & $\begin{array}{c}5 \\
7 \\
5 \\
18\end{array}$ & $\begin{array}{l}14 \\
20 \\
14 \\
52\end{array}$ \\
\hline $\begin{array}{l}\text { ¿Cuándo iniciar los CPP? } \\
\text { En el momento del diagnóstico } \\
\text { En los últimos } 6 \text { meses de vida } \\
\text { Cuando presente dolor refractario } \\
\text { Cuando la familia lo solicite } \\
\text { No precisa recibiendo tratamiento activo }\end{array}$ & $\begin{array}{c}32 \\
1 \\
0 \\
2 \\
0\end{array}$ & $\begin{array}{l}91 \\
\frac{3}{6} \\
\frac{-}{-}\end{array}$ \\
\hline $\begin{array}{l}\text { ¿Dónde debe morir un niño? } \\
\text { En su domicilio } \\
\text { En el hospital }\end{array}$ & $\begin{array}{c}33 \\
1\end{array}$ & $\begin{array}{c}97 \\
3\end{array}$ \\
\hline $\begin{array}{l}\text { Grupo mayoritario en las unidades de CPP } \\
\text { Oncológicos } \\
\text { No oncológicos }\end{array}$ & $\begin{array}{l}25 \\
10\end{array}$ & $\begin{array}{l}71 \\
29\end{array}$ \\
\hline $\begin{array}{l}\text { ¿Atiende algún paciente subsidiario de CPP? } \\
\text { Sí } \\
\text { No }\end{array}$ & $\begin{array}{l}13 \\
22\end{array}$ & $\begin{array}{l}37 \\
63\end{array}$ \\
\hline $\begin{array}{l}\text { ¿Formación previa en CPP? } \\
\text { Sí } \\
\text { No }\end{array}$ & $\begin{array}{c}8 \\
27\end{array}$ & $\begin{array}{l}23 \\
77\end{array}$ \\
\hline $\begin{array}{l}\text { ¿Considera su formación adecuada? } \\
\text { Muy adecuada } \\
\text { Adecuada } \\
\text { Poco adecuada } \\
\text { Nada adecuada }\end{array}$ & $\begin{array}{c}0 \\
0 \\
16 \\
19\end{array}$ & $\begin{array}{l}- \\
46 \\
54\end{array}$ \\
\hline $\begin{array}{l}\text { ¿Interés en ampliar sus conocimientos? } \\
\text { Sí } \\
\text { No }\end{array}$ & $\begin{array}{c}33 \\
1\end{array}$ & $\begin{array}{c}97 \\
3\end{array}$ \\
\hline $\begin{array}{l}\text { Barreras identificadas del escaso papel en atención primaria } \\
\text { Falta de formación } \\
\text { Escasa disponibilidad y tiempo } \\
\text { Falta de un equipo de apoyo } \\
\text { Todas las anteriores }\end{array}$ & $\begin{array}{c}9 \\
2 \\
3 \\
15\end{array}$ & $\begin{array}{c}31 \\
7 \\
10 \\
52\end{array}$ \\
\hline $\begin{array}{l}\text { ¿Atendería pacientes en la fase final de su enfermedad? } \\
\text { Sí } \\
\text { No }\end{array}$ & $\begin{array}{c}29 \\
5\end{array}$ & $\begin{array}{l}85 \\
15\end{array}$ \\
\hline $\begin{array}{l}\text { ¿Existe unidad de CPP en su comunidad? } \\
\text { Sí } \\
\text { No } \\
\text { No lo sé }\end{array}$ & $\begin{array}{c}4 \\
18 \\
13\end{array}$ & $\begin{array}{l}11 \\
52 \\
37\end{array}$ \\
\hline $\begin{array}{l}\text { ¿Cree que sería necesaria? } \\
\text { Muy necesaria } \\
\text { Bastante necesaria } \\
\text { Poco necesaria } \\
\text { Nada necesaria }\end{array}$ & $\begin{array}{c}22 \\
11 \\
1 \\
0\end{array}$ & $\begin{array}{c}65 \\
32 \\
3 \\
-\end{array}$ \\
\hline
\end{tabular}

CPP: cuidados paliativos pediátricos.

*Mediana (rango).

consideró que «es proporcionar bienestar, ayuda, incurables o amenazantes para la vida». Otros calidad de vida, cuidado y atención integral, alivio del centraban los mismos cuidados en pacientes en fase dolor y del sufrimiento a pacientes con enfermedades terminal o al final de la vida. 
El $91 \%$ de los pediatras consideró que se deberían iniciar los cuidados paliativos en el momento del diagnóstico de una enfermedad incurable o amenazante para la vida. Dos personas señalaron que se deberían iniciar cuando la familia los solicitara, y una, en los últimos 6 meses de vida del paciente.

El $97 \%$ de los encuestados afirmaron que el mejor lugar donde debería fallecer un niño era en su domicilio, siempre que los cuidados fueran los adecuados.

Por otro lado, el $71 \%$ de los participantes consideraron que los pacientes oncológicos son los mayoritariamente atendidos en las unidades de CPP.

En cuanto a la oportunidad de trabajo con pacientes con necesidades de cuidados paliativos, el $63 \%$ de los participantes no atendía ningún paciente en su cupo que pudiera ser subsidiario de recibir estos cuidados. Solo ocho personas habían recibido anteriormente algún tipo de formación sobre CPP. El 100\% de los encuestados consideró no poseer una formación adecuada en CPP: el 54\% dijo tener una formación nada adecuada, y el $46 \%$, poco adecuada. Cabe señalar que el $97 \%$ manifestó tener interés por ampliar sus conocimientos en este ámbito.

En cuanto a las barreras identificadas como principales causas del escaso papel de la atención primaria en este tipo de pacientes, el $31 \%$ se centró en la falta de formación, el 10\%, en la falta de un equipo de apoyo y el $7 \%$ en la escasa disponibilidad y la falta de tiempo. El $52 \%$ consideró que el problema se centraba en las tres afirmaciones anteriores.

Al preguntar a los participantes si atenderían a pacientes en la fase final de su enfermedad en caso de que mejorara alguno de los aspectos que consideraban necesarios, solo cinco personas se negarían a participar en la atención.

Llama la atención que cuatro de los participantes creían que, en el momento del estudio, existía una unidad de CPP en la región, y que 13 personas desconocían si existía o no, cuando la realidad era que, en ese momento, no se disponía de ningún tipo de unidad específica en la comunidad.

Por último, la gran mayoría (97\%) afirmó que creían necesaria una unidad de CPP en la comunidad; de ellos, el $65 \%$ la consideró indispensable. Solo una persona manifestó que dicha unidad sería poco necesaria.

\section{Discusión}

Estamos preparados para el estudio de la vida, pero no para el de la muerte. El concepto de muerte y lo que esta conlleva ha cambiado a lo largo de la historia, de tal forma que, actualmente, la muerte se vive como un fracaso en el ámbito sanitario. Se olvidan las necesidades básicas del paciente; no solo las físicas, sino también las psicológicas y espirituales.

Con los avances tecnológicos actuales en prevención, diagnóstico y tratamiento de las enfermedades es difícil asumir la no curación, y con mayor razón cuando ocurre en niños.

La muerte de un niño no es un acontecimiento esperable es un hecho que invierte el orden natural de la vida. Sin embargo, la dura realidad es que los niños también mueren.

Reconocer la muerte como un proceso permite acompañar al paciente y a su familia en el camino al fallecimiento. Identificar las diferentes fases y las necesidades del paciente posibilita la adecuación del esfuerzo terapéutico para la obtención de su mayor bienestar.

En España, entre 2002 y 2011 fallecieron aproximadamente 3300 niños por año (datos del Instituto Nacional de Estadística). El $60 \%$ de las muertes pediátricas se produjeron por causas previsibles; es decir, por enfermedades que podrían ser susceptibles de cuidados paliativos ${ }^{9}$. Un tercio de ellas son enfermedades oncológicas, y el resto son de tipo neurológico o metabólico, entre otros.

La OMS mantiene cuatro aspectos fundamentales en los que deberían apoyarse los cuidados al final de la vida: políticas sanitarias adecuadas, disponibilidad y conocimiento en el manejo de opiáceos, formación adecuada de los trabajadores sanitarios y puesta en marcha de servicios de cuidados paliativos en todos los niveles.

Es responsabilidad ética de los sistemas de salud proporcionar cuidados paliativos y que los profesionales sanitarios tengan la obligación ética de aliviar el dolor y el sufrimiento, ya sea de orden físico, psicosocial o espiritual ${ }^{10}$.

Los CPP no son exactamente cuidados paliativos similares a los de adultos aplicados en niños, sino que se diferencian en varios puntos que, a la vez, son limitaciones para su desarrollo ${ }^{11}$. Entre estas diferencias se encuentran las siguientes:

- Menor número de casos (en el Principado de Asturias, la población mayoritaria es de edad avanzada, con una baja tasa de natalidad).

- Amplia dispersión geográfica (en el Principado de Asturias, la población se concentra en la zona centro, dejando las alas de la comunidad más despobladas). - Aspectos cognitivos y madurativos propios del desarrollo del niño. 
- Tratamiento farmacológico limitado para su uso en pacientes pediátricos.

- Falta de formación profesional.

- Impacto social y falta de cultura con respecto al cuidado de los niños que van a morir.

Existe un acuerdo tajante entre los pediatras de atención primaria del Principado de Asturias en cuanto a que no poseen formación adecuada en CPP. Quizás esta falta de formación pueda influir en la dificultad de reconocimiento de los pacientes subsidiarios de CPP, y en que el número de ellos en realidad sea mayor.

Este escaso nivel formativo y de conocimientos del personal, evidenciado en el presente estudio, coincide con otros datos referidos en la literatura médica consultada.

En el trabajo de Medina, et al. ${ }^{12}$ solo el $7 \%$ del personal de salud encuestado de tres niveles de atención médica obtuvo resultados aceptables en conocimientos totales sobre CPP ${ }^{12}$.

En el reporte de Astray ${ }^{13}$, que encuestó a pediatras de atención primaria de un área de la comunidad de Madrid, el $50.8 \%$ afirmó necesitar preparación y el $55.4 \%$ refirió no haber leído ningún artículo sobre CPP en los últimos 5 años. Además, destacaba el dato de que únicamente el 35\% conocía la existencia de la única unidad de CPP de la comunidad.

En una encuesta respecto a la formación en medicina paliativa realizada a residentes de una de las escuelas de posgrado más importantes de Chile, se demostró que esta era insuficiente (el $76 \%$ de los encuestados no tuvieron formación en cuidados paliativos y la mitad de los encuestados consideró que su preparación era menor del $25 \%$ de lo considerado como necesario) ${ }^{14}$. A pesar de ello, destacan el deseo y la disposición a mejorar esta situación, ya que el $97 \%$ de los encuestados estaban interesados en ampliar sus conocimientos, aunque no todos los pediatras estaban dispuestos a proporcionar cuidados al final de la vida en caso de que fuera necesario.

Las causas más probables de este mínimo rechazo seguramente son las reflejadas como barreras principales en el escaso papel del pediatra de atención primaria en CPP: la falta de formación aunada a la falta de un equipo de apoyo, la escasa disponibilidad y la falta de tiempo (sobrecarga de las consultas de atención primaria). En el presente estudio, hasta el $52 \%$ de los participantes identificaron las tres causas como parte del problema.

En un estudio, los pediatras generales que proporcionaban CPP en una zona rural de Alemania manifestaron la necesidad de apoyo de un equipo especializado, incluyendo las 24 horas del día y la intensificación de programas formativos ${ }^{15}$.

En el Principado de Asturias, las diferentes unidades de hospitalización domiciliaria y los equipos de apoyo de cuidados paliativos de cada área sanitaria (dirigidos principalmente a adultos) determinan si se requiere atención pediátrica domiciliaria. Este hecho es desconocido por gran parte de los pediatras, ya que hasta el 37\% no sabía si existía una unidad específica de CPP y casi el $12 \%$ creía que sí se contaba con ella.

Al parecer, de forma casi unánime, el $97 \%$ de los participantes, en mayor o menor medida, creen necesario el establecimiento de una unidad de CPP en la comunidad.

La capacidad del médico para afrontar la comunicación de malas noticias, diagnósticos inesperados, fracaso de tratamientos o la muerte de un paciente, la mayoría de las veces depende de los propios recursos personales y de la experiencia previa, debido a la falta de una formación específica.

La falta de formación en el ámbito de la comunicación de situaciones difíciles puede tener un efecto devastador en el paciente y su familia. La devastación psicológica causada por un diagnóstico comunicado de forma inadecuada puede ser no menos grave que los errores quirúrgicos 0 de medicación ${ }^{16}$. También la falta de seguridad, comodidad y control de estas situaciones puede afectar a los propios profesionales de la salud, tanto en el ámbito laboral como en el personal (agotamiento emocional, ansiedad o tristeza, entre otros).

Por estudios previos se conoce la efectividad de la formación de los pediatras en CPP. Después de la formación en cuidados paliativos, los médicos poseen más confianza en el manejo de varias situaciones, como apoyo emocional, conocimiento personal, habilidades y comunicación ${ }^{17}$.

La mayoría de los profesionales defienden un mínimo de adiestramiento, experiencia, conocimiento y competencias en todas las áreas de CPP, principalmente en la comunicación con el paciente y la familia, y en el manejo del dolor ${ }^{18}$, aunque también en la discusión de pronósticos y en cómo proporcionar malas noticias ${ }^{19}$. La humanización, los cuidados y el acompañamiento son cualidades que deberían estar unidas y que son innatas de la medicina en general.

Por todo ello, sería absolutamente necesaria la implantación de una visión de la medicina desde el punto de vista no solamente de curación, investigación y promoción de la salud, sino también del 
alivio del sufrimiento, el acompañamiento y la calidad de vida, en las facultades de medicina y enfermería (que son la base del conocimiento y la formación de cualquier profesional sanitario). Además, debe considerarse la inclusión de los conocimientos sobre enfermedades potencialmente mortales, sus síntomas, el alivio del sufrimiento, las habilidades de comunicación y los procedimientos de decisión ética clínica en los programas de formación de residentes de pediatría 20,21 .

Como limitación del presente estudio es importante destacar la baja participación. De los 85 pediatras de atención primaria a los que se envió la encuesta, solo la contestaron 35 (41.2\%). Esta baja participación puede deberse a varios factores:

- El escaso papel de los CPP en la comunidad y, por lo tanto, el desconocimiento del tema.

- Pocos pacientes subsidiarios de estos cuidados en atención primaria y, por consiguiente, la escasa visión del paciente paliativo y de la importancia de los cuidados de calidad.

- Poca disponibilidad y tiempo en las consultas de atención primaria saturadas.

De cualquier forma, aunque la participación hubiera sido mayor, probablemente las respuestas habrían sido semejantes, dada la tendencia de las respuestas obtenidas.

Como conclusiones, se puede afirmar que, por lo general, los pediatras de atención primaria del Principado de Asturias conocen parcialmente qué son los CPP y cuál es su cometido, y son conscientes de sus propias deficiencias en cuanto a su formación en este ámbito, pero también son capaces de identificar cuáles son las principales causas de esta situación y, consecuentemente, cómo modificarla.

Se necesita una mayor formación en CPP para conseguir, entre otras cosas, que el pediatra de atención primaria se convierta en una figura clave para proporcionar cuidados básicos a este tipo de pacientes.

Dada la buena disposición de los profesionales, con deseos de aprendizaje y mejora en su formación, la sanidad pública debería realizar un esfuerzo para mejorar la formación en CPP.

\section{Responsabilidades éticas}

Protección de personas y animales. Los autores declaran que para esta investigación no se han realizado experimentos en seres humanos ni en animales.
Confidencialidad de los datos. Los autores declaran que han seguido los protocolos de su centro de trabajo sobre la publicación de datos de pacientes.

Derecho a la privacidad y consentimiento informado. Los autores han obtenido el consentimiento informado de los pacientes o sujetos referidos en el artículo. Este documento obra en poder del autor de correspondencia.

\section{Conflicto de intereses}

Los autores declaran no tener ningún conflicto de intereses.

\section{Financiamiento}

$$
\text { Ninguno. }
$$

\section{Agradecimientos}

A todos aquellos pediatras participantes que obsequiaron parte de su tiempo para contestar el cuestionario.

\section{Bibliografía}

1. World Health Organization. Definition of palliative care. 2018. Disponible en: http://www.who.int/cancer/palliative/definition/en/.

2. Grupo de Trabajo de Cuidados Paliativos Pediátricos de la Asociación Europea de Cuidados Paliativos (EAPC). Cuidados paliativos para lactantes, niños y jóvenes: los hechos. Roma: Fundación Maruzza Lefebvre d'Ovidio Onlus; 2009.

3. Santos HP, Losa FV, Huidobro LB. Cuidados paliativos en pediatría. Form Act Pediatr Aten Prim. 2017;10:109-14.

4. Organización Mundial de la Salud. Primer atlas mundial de las necesidades de cuidados paliativos no atendidas. 2014. Disponible en: https://www.who.int/mediacentre/news/releases/2014/palliative-care-20140128/es/.

5. Info paliativos. SECPAL reclama que todas las CCAA tengan al menos una unidad de cuidados paliativos pediátricos para atender a todos los menores que los necesitan. Madrid; 2020.

6. Sociedad Española de Cuidados Paliativos (SECPAL). Análisis y evaluación de los recursos de cuidados paliativos de España. Madrid: Sociedad Española de Cuidados Paliativos (SECPAL); 2016.

7. Villalba NJ. Afrontamiento de la muerte de un niño: los cuidados pediátricos paliativos desde la atención primaria. Rev Pediatr Aten Primaria. 2015;17:171-83.

8. Portal de Salud del Principado de Asturias. Estrategia de cuidados paliativos para Asturias. Oviedo: Consejería de Sanidad, Principado de Asturias; 2009.

9. Universidad Internacional de la Rioja. Tema 3. Marco conceptual: Epidemiología, principios y atención al final de la vida. Máster en Cuidados Paliativos Pediátricos. La Rioja: Universidad Internacional de La Rioja; 2018.

10. Organización Mundial de la Salud. $67^{\circ}$ Asamblea Mundial de la Salud. Fortalecimiento de los cuidados paliativos como parte del tratamiento integral a lo largo de la vida. 2014.

11. Calleja GML, Rus PM, Martino AR, Monleón LM, Conejo MD, Ruiz-Falcó RML. Cuestionario sobre cuidados paliativos a neuropediatras. Neurología. 2012;27:277-83.

12. Medina ZLE, de la Cruz CAM, Sánchez SME, González-Pedraza AA Nivel de conocimientos del personal de salud sobre cuidados paliativos. Rev Esp Med Quir. 2012;17:109-14.

13. Astray SMA. Encuesta sobre cuidados paliativos a pediatras de atención primaria en un área sanitaria de Madrid. Rev Pediatr Aten Primaria. 2010;12:33-40. 
14. Vial CP, Ibáñez LP, Umaña VA, Reyes DMM, Viviani P, Nervi OF. Autoevaluación sobre formación en medicina paliativa en una cohorte de residentes. Rev Med Chile. 2004; 132:445-52.

15. Kremeike K, Eulitz N, Jünger $S$, Sander A, Geraedts M, Reinhardt D. Paediatric palliative home care in areas of Germany with low population density and long distances: a questionnaire survey with general paediatricians. BMC Res Notes. 2012;5:1-8.

16. Barriuso Feijoo J, González Barón M. Comunicación e información. Tratado de medicina paliativa y tratamiento de soporte del paciente con cáncer. Madrid: Médica Panamericana; 2007. p. 61-4.
17. Peng NH, Lee CH, Lee MC, Huang LC, Chang YC, DeSwarte-Wallace J. Effectiveness of pediatric palliative care education on pediatric clinicians. West J Nurs Res. 2017;39:1624-38.

18. Kolarik RC, Walker G, Arnold RM. Pediatric resident education in palliative care: a needs assessment. Pediatrics. 2006;117:1949-54.

19. Michelson KN, Ryan AD, Jovanovic B, Frader J. Pediatric residents 'and feUlows' perspectives on palliative care education. J Palliat Med 2009:12:451-7.

20. Martino AR. El proceso de morir en el niño y en el adolescente. Pediatr Integral. 2007;11:926-34.

21. Liben S, Papadatou D, Wolfe, J. Paediatric palliative care: challenges and emerging ideas. Lancet. 2008;371:852-64. 